\title{
Entanglement creation with negative index metamaterials
}

\author{
Michael Siomau ${ }^{1}$, Ali A. Kamli ${ }^{1}$, Sergey A. Moiseev ${ }^{2}$ and Barry C. Sanders ${ }^{3}$ \\ ${ }^{1}$ Physics Department, Jazan University, P.O. Box 114, 45142 Jazan, Kingdom of Saudi Arabia \\ ${ }^{2}$ Kazan Physical-Technical Institute of Russian Academy of Science, 420029 Kazan, Russia and \\ ${ }^{3}$ Institute for Quantum Information Science, University of Calgary, Alberta T2N 1N4, Canada
}

(Dated: November 20, 2018)

\begin{abstract}
We propose a scheme for creating of a maximally entangled state comprising two field quanta. In our scheme, two weak light fields, which are initially prepared in either coherent or polarization states, interact with a composite medium near an interface between a dielectric and a negative index metamaterial. Such interaction leads to a large Kerr nonlinearity, reduction of the group velocity of the light and significant confinement of the light fields while simultaneously avoiding amplitude losses of the incoming radiation. All these considerations make our scheme efficient.
\end{abstract}

PACS numbers: 03.67.Bg, 42.50.Dv, 42.50.Gy, 03.67.Hk

Entanglement is an essential feature of quantum theory, which manifests impressive advantages of recently established quantum technologies over their classical counterpart [1, 2]. Variety of physical systems, starting from atoms, electrons and photons [3] and ending with sophisticated molecules and even living organisms [4], can exhibit quantum entanglement. Among these systems, photons, the quanta of electromagnetic field, take privileged position because of their exceptional properties. Photons can be easily generated and measured and can carry information over long distances being resistant to detrimental effect of decoherence [5]. At the same time, photons do not directly interact with each other, what makes difficult to prepare them in entangled states.

A conventional way to create entanglement of light quanta is through their interaction with nonlinear (Kerr) medium, which have intensity-dependent refractive index [6]. In natural medium, however, Kerr nonlinearity is very small. Therefore, to achieve significant entanglement of photons, one has to increase both intensity of the field pulses and interaction time with the medium. Such actions may not be always possible in practice, because of diffraction and finite size of the medium, and are very unlikely from the viewpoint of applications [5, 7], where weak light fields (i.e. of energy of a single photon) are desired.

In 2000, a pioneering proposal to "design" material exhibiting strong nonlinear interaction at the single-photon level was made by Lukin and Imamoğlu [8]. This idea stimulated a number of theoretical investigations 9 [ 11$]$ as well as experiments [12 14], to name just a few. However, despite these remarkable results in achieving large Kerr nonlinearity, efficient creation of entanglement at the low energy limit remains challenging in many aspects [15, 16].

In this work we suggest a scheme in which initially uncorrelated states of light field become entangled due to their interaction with a medium near an interface between a dielectric and a negative index metamaterial. The medium of interest consists of a dielectric (which has a layer of thickness $z_{0}$ doped with six level atoms [9]) and a metamaterial placed together, as shown in Fig. 1] Due to interaction with the medium, an incident light beam creates a spatially confined surface polariton [17] which propagates along the interface with substantially reduced group velocity $v_{g}<c$. Although in natural mediums a surface polariton undergos slashing amplitude loss, specific design of the medium makes possible to suppress losses significantly in a narrow frequency bandwidth of the incoming light [19]. Placing the layer of six level atoms near the interface allows us to establish double electromagnetically induced transparency [20] (i.e. for two incoming pulses simultaneously) and, at the same time, create large Kerr nonlinearity [9]. All mentioned factors contribute to an efficient nonlinear interaction between the two surface polaritons in the medium. Such interaction makes possible a mutual $\pi$-phase shift between the polaritons which leads to entanglement of the light fields.

Ignoring presence of the atomic layer near the interface, the process of interaction between the light fields and the medium can be considered from the viewpoint of classical electrodynamics. Macroscopic properties of the material can be characterized with electric permittivity

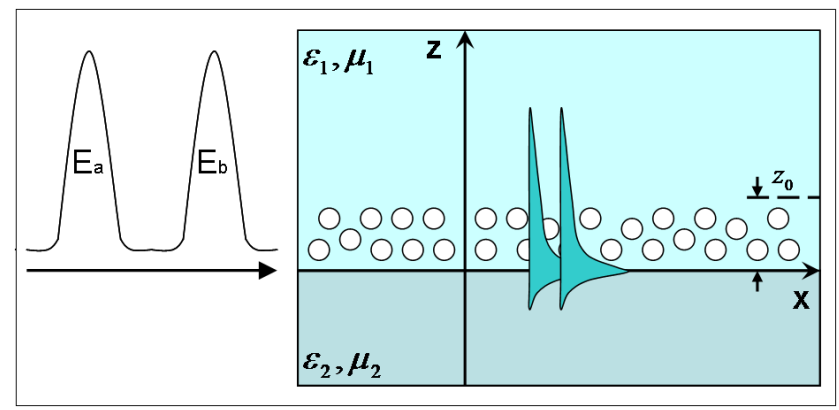

FIG. 1: (Color online) Two weak light pulses create two surface polaritons near the interface between the dielectric $z>0$ and the metamaterial $z<0$. While electromagnetically induced transparency is established for both polaritons simultaneously, they will propagate along the interface with small group velocities $v_{g}<c$ and interact nonlinearly with each other. 
$\varepsilon$ and magnetic permeability $\mu$. For a dielectric, these parameters are strictly positive, while both of them may be simultaneously negative for a metamaterial 21]. Let us assume that the dielectric has constant homogeneous parameters $\varepsilon_{1}$ and $\mu_{1}$, while parameters $\varepsilon_{2}$ and $\mu_{2}$ are frequency-dependent for the metamaterial and are given by [19]

$$
\begin{aligned}
& \varepsilon_{2}(\omega)=\varepsilon_{\infty}-\frac{\omega_{e}^{2}}{\omega\left(\omega+i \gamma_{e}\right)}, \\
& \mu_{2}(\omega)=\mu_{\infty}-\frac{\omega_{m}^{2}}{\omega\left(\omega+i \gamma_{m}\right)},
\end{aligned}
$$

where $\omega_{e}$ and $\omega_{m}$ are electric and magnetic plasma frequencies, $\gamma_{e}$ and $\gamma_{m}$ are corresponding (empiric) decay rates and $\varepsilon_{\infty}$ and $\mu_{\infty}$ are background constants [17. Here we have chosen the simplest (Drude-like) model for magnetic permeability $\mu_{2}(\omega)$. This model is known to be adequate in the optical region [17, 18], although more sophisticated models can be taken into consideration [22].

Electromagnetic field of the surface polaritons can be found form Maxwell equations with boundary conditions for $\varepsilon_{i}$ and $\mu_{i}(i=1,2)$. Since the permittivity and the permeability (11) may be simultaneously negative, both transverse magnetic (TM) and transverse electric (TE) polarizations of the electromagnetic field may exist in the medium. Natural mediums, in contrast, support only TM polarization [17]. To be specific, we shall later focus on the TM waves. The wave vector of the electromagnetic field in the medium is given by the dispersion relation as follows

$$
K(\omega)=\frac{\omega}{c} \sqrt{\varepsilon_{1} \varepsilon_{2} \frac{\varepsilon_{2} \mu_{1}-\varepsilon_{1} \mu_{2}}{\varepsilon_{2}^{2}-\varepsilon_{1}^{2}}} .
$$

The real part of this expression gives dispersion of the field, while its imaginary part stands for absorbtion loss.

It has been shown that absorbtion loss can be completely suppressed in a narrow frequency bandwidth due to destructive interference of electric and magnetic absorbtion responses of the medium [19]. For example, taking $\varepsilon_{1}=1.3$ and $\mu_{1}=1$ and $\omega_{e}=1.37 \times 10^{16} \mathrm{~s}^{-1}, \gamma_{e}=$ $2.73 \times 10^{13} \mathrm{~s}^{-1}$ (as for Ag) and assuming $\omega_{m}=10^{15} \mathrm{~s}^{-1}$, $\gamma_{m}=10^{12} \mathrm{~s}^{-1}, \varepsilon_{\infty}=5$ and $\mu_{\infty}=5$, one can see that absorbtion loss $\operatorname{Im}[K(\omega)]$ vanishes for $\omega_{0} \approx 4.4 \times 10^{14} \mathrm{~s}^{-1}=$ $440 \mathrm{THz}$, what corresponds to red light of visible spectrum. It is important to note that metamaterials with negative refractive index have been observed in the red region of visible spectrum [21]. More details about the dispersion relation (21) and possible parameters of the medium can be found in Ref. [19] and references therein.

We are now at the position to consider the interaction between the surface polariton light fields and the medium quantum mechanically. Electric field of each of the surface polaritons can be quantized near the surface and written in the plane wave expansion as 23.

$$
\mathbf{E}(\mathbf{r}, t)=\int \mathrm{d} k\left[\mathbf{E}_{0}(k) a(k) e^{i k x-\omega t}+\text { H.c. }\right] .
$$

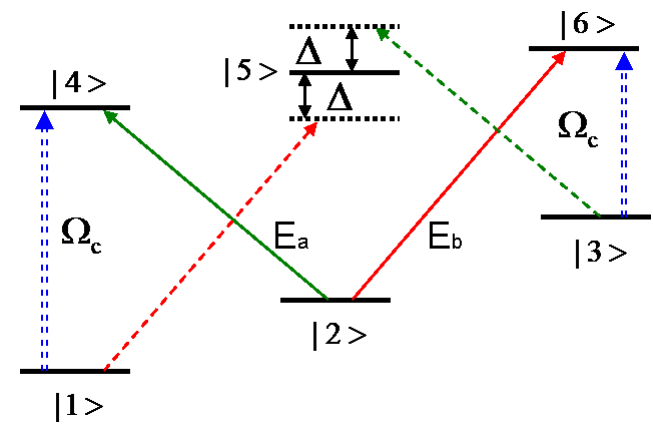

FIG. 2: (Color online) Configuration of a six level atom for creating double electromagnetically induced transparency for two weak fields $E_{a}$ and $E_{b}$. Here, the fields $E_{a}$ and $E_{b}$ are coupled with transitions $|2\rangle \rightarrow|4\rangle$ and $|2\rangle \rightarrow|6\rangle$ resonantly and with transitions $|3\rangle \rightarrow|5\rangle$ and $|1\rangle \rightarrow|5\rangle$ off resonantly with detuning $\Delta$. Two classical control fields drive transitions $|1\rangle \rightarrow|4\rangle$ and $|3\rangle \rightarrow|6\rangle[9]$.

Here we introduced $k \equiv \operatorname{Re}[K(\omega)]$, taking into account that the wave vector is approximated by its real part $k(\omega) \approx \operatorname{Re}[K(\omega)]$ in the low loss frequency range. The amplitude $\mathbf{E}_{0}(k)$ can be found from the requirement that it should obey the field Hamiltonian $\mathrm{H}_{F}=1 / 2 \int d^{3} r[\tilde{\varepsilon}<$ $|\mathbf{E}|^{2}>+\tilde{\mu}<|\mathbf{H}|^{2}>$ ] in a dispersive lossless medium [22]. It is important to note that our quantization procedure is applicable only in a narrow frequency bandwidth where the losses are low. In general case, the quantization of electromagnetic field in dispersive and absorptive media is a much more complicated task [24 26].

The quantized polariton fields exhibit a remarkable property of confinement along $z$ direction. The confinement can be quantified as $\xi(\omega)=1 / \operatorname{Re}\left[k^{\perp}(\omega)\right]$, where $k^{\perp}(\omega)=\sqrt{K^{2}(\omega)-\omega^{2} \varepsilon_{1} \mu_{1} / c^{2}}$ is the normal component of the real part of the wave vector (2). This property of the quantized fields ensure interaction of the polaritons with the six level atoms embedded into the dielectric and defines effective thickness of the atomic layer $\xi(\omega) \approx z_{0}$ in practice. The reason of injection the six level atoms near the dielectric-metamaterial interface, is that such atomic system has been shown to cause the effect of double electromagnetically induced transparency simultaneously exhibiting symmetric nonlinearity for two incoming pulses [9]. The energy levels of the system are shown schematically in Fig. 2. The model of a six level atom can be practically realized on the rubidium isotope ${ }^{87} \mathrm{Rb}[9]$, for example.

The interaction of the surface polaritons and the six level atoms can be modeled with electric dipole hamiltonian $\mathrm{H}_{E D}=-\sum \mathbf{d}_{i} \cdot \mathbf{E}\left(\mathbf{r}_{i}\right)$, where $\mathbf{E}\left(\mathbf{r}_{i}\right)$ is the electric field (3) of the surface polaritons, $\mathbf{r}_{i}$ the position of the atoms and the summation is to be done over all atoms in the interaction volume [22].

Because of the symmetry of the atomic levels structure the two surface polaritons propagate in the medium with equal group velocities $v_{a}=v_{b} \equiv v_{g}[9]$. Dynamics of 


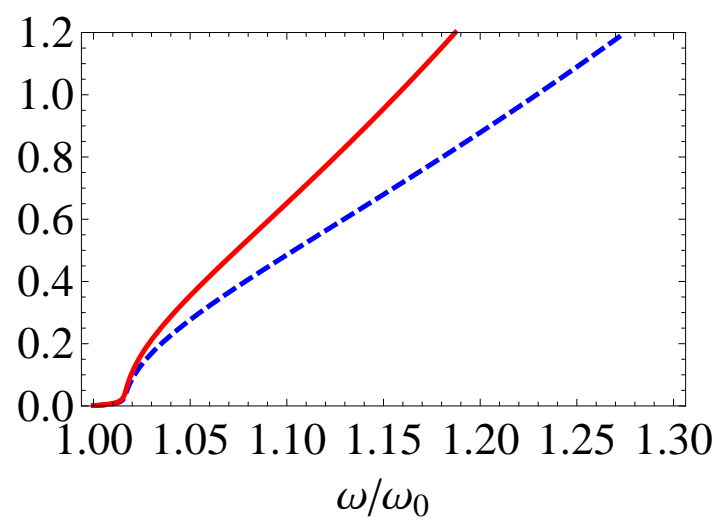

FIG. 3: (Color online) Kerr coefficient $\chi \times 10^{4}$ (dashed blue) and corresponding mutual phase shift $\phi$ in units $\pi$ (solid red) as functions of the field frequency $\omega / \omega_{0}$.

the surface polariton field operators can be obtained in Heisenberg picture by solving corresponding set of equations

$$
\left(\frac{1}{v_{g}} \frac{\partial}{\partial t}+\frac{\partial}{\partial x}\right) \mathbf{E}_{n}(\mathbf{r}, t)=i \chi I_{m} \mathbf{E}_{n}(\mathbf{r}, t)
$$

where the adiabatic approximation has been used to ignore time derivatives of higher order [22]. Here $n, m=$ $a, b(n \neq m), I_{m}=\left|\mathbf{E}_{m}(\mathbf{r}, t)\right|^{2}$ and $\chi$ is Kerr coefficient given by

$$
\chi=\frac{2 \pi n z_{0} f\left[\left(k_{a}+k_{b}-k_{c}\right) z_{0}\right]}{\hbar^{4} v_{g}\left|\Omega_{c}\right|^{2} \Delta}<\left|\mathbf{d}_{24} \mathbf{E}_{a}\right|^{2}\left|\mathbf{d}_{26} \mathbf{E}_{b}\right|^{2}>,
$$

where $n$ is atomic density, $z_{0}$ is thickness of the atomic layer, $f[x] \equiv\left(e^{-x} \sinh x\right) / x, k_{a}$ and $k_{b}$ are the real parts of the polaritons wave numbers, $k_{c}$ and $\Omega_{c}$ are the wave number and the Rabi frequency of the driving field, $v_{g}$ is group velocity of the polaritons ignoring the atomic layer, $\Delta$ stands for spectral detuning, $\mathbf{d}_{24}$ and $\mathbf{d}_{26}$ give atomic dipole moments of the corresponding transitions, $\mathbf{E}_{a}$ and $\mathbf{E}_{b}$ are electric field operators of the polaritons and $\langle\ldots\rangle$ denotes averaging over orientation of the dipole moments. Typical atomic density in a gas is $2 \times 10^{14} \mathrm{~cm}^{-3}$. To establish double electromagnetically induced transparency in ${ }^{87} \mathrm{Rb}$, Rabi frequency of the control field is to be $\Omega_{c}=1 \mathrm{MHz}$, the transition wavelength is $780 \mathrm{~nm}$, the detuning is $\Delta=1.4 \mathrm{MHz}$ and the dipole moments are about $5 e a_{0}$, where $e$ is the electron charge $a_{0}$ is the Bohr radius. Assuming the thickness of the atomic layer $z_{0}=2 \mu \mathrm{m}$, we obtain Kerr nonlinearity as displayed in Fig. 3 ,

Although the Kerr nonlinearity $\chi$ is of order of $10^{-4}$, a significant mutual phase shift of order of unity can be achieved between the surface polaritons. The mutual phase shift is given by $\phi=\chi \omega L / v_{g}$, where $\omega$ is the light frequency, $L$ is the length of interaction in the medium and $v_{g}$ is the group velocity of the light in the medium ignoring the layer of the five level atoms. For chosen parameters of the medium $v_{g} \approx 0.4 c$ and assuming $L=1 \mathrm{~mm}$, the mutual phase shift is shown in Fig. 3 . The surface polaritons receive a mutual phase shift of order of $\pi$ at frequency $\omega_{\pi} \approx 1.24 \omega_{0}=545 \mathrm{THz}$ (green light) which is close to the no-loss frequency $\omega_{0}$.

Here we would like to point out that, because of the symmetry of the levels of the six level atom, the refractive index of the medium is exactly the same for two surface polaritons. That is why the polaritons propagate in the medium with equal group velocities and experience identical nonlinearity. Alternatively, five level atoms [10] can be placed near dielectric-metamaterial interface [27]. In this case, two surface polaritons propagate in the medium with different group velocities and experience different nonlinearity in the double electromagnetically induced transparency regime. Latter scheme is best suitable to achieve a uniform cross-phase modulation [28].

The mutual (symmetric) phase shift can be used to create entanglement between initially uncorrelated field modes. For single-mode incident fields, the interaction of the surface polaritons in the (Kerr) medium can be described with the help of an effective Hamiltonian

$$
\mathrm{H}_{\mathrm{eff}}=\hbar \chi a^{\dagger} a b^{\dagger} b,
$$

where $a^{\dagger}, b^{\dagger}$ and $a, b$ are creation and annihilation operators of the field modes of the two polaritons. Time evolution of these operators is initiated by the unitary transformation $U(t)=\exp \left(-i \phi a^{\dagger} a b^{\dagger} b\right)$ and is given by

$$
a(t)=e^{-i \phi b^{\dagger} b} a(0), \quad b(t)=e^{-i \phi a^{\dagger} a} b(0) .
$$

If the initial states of the incident fields are uncorrelated single-mode coherent states $|\alpha\rangle$ and $|\beta\rangle$ [23], the final state $|\psi(t)\rangle_{a b}$ after the interaction can be written in the Fock basis as 29]

$$
|\psi(t)\rangle_{a b}=e^{-\frac{|\beta|^{2}}{2}} \sum_{n=0}^{\infty} \frac{\beta^{n}}{\sqrt{n !}}\left|\alpha e^{-i \phi n}\right\rangle_{a} \otimes|n\rangle_{b},
$$

where the dynamics of the creation and annihilation operators (7) has been taken into account. Assuming $\phi=\pi$ and decomposing the sum in Eq. (8) over odd and even values of the index $n$, we obtain the following form of the final state

$$
|\psi\rangle_{a b}^{f}=\frac{1}{2}\left(|\alpha\rangle_{a}\left(|\beta\rangle_{b}+|-\beta\rangle_{b}\right)+|-\alpha\rangle_{a}\left(|\beta\rangle_{b}-|-\beta\rangle_{b}\right)\right) .
$$

This state is local unitary equivalent to the entangled state $\left(|\alpha\rangle_{a}|\beta\rangle_{b}+|-\alpha\rangle_{a}|-\beta\rangle_{b}\right) / \sqrt{N}$ [29], where $N=2-$ $2 \exp \left(-2|\alpha|^{2}-2|\beta|^{2}\right)$, which is known to preserve exactly one e(ntangled )bit of quantum information [30].

In contrast to our assumption above, the initial states $|\psi\rangle_{a}$ and $|\psi\rangle_{b}$ of the incident field can be also assumed to be polarization states of photons. In this case, entanglement of the field modes can be achieved, for example, with the help of Nemoto-Munro protocol [31]. To understand this protocol better, let us assume that the incident field $a$ is prepared into a superposition of vacuum and a 
single photon states and is given by $|\psi\rangle_{a}=c_{0}|0\rangle+c_{1}|1\rangle$ in Fock basis. The second incident field is prepared into a coherent state $|\alpha\rangle_{b}$. When the two fields interact with the media, the resulting state of the fields is given by

$$
U(t)|\psi\rangle_{a}|\alpha\rangle_{b}=c_{0}|0\rangle|\alpha\rangle_{b}+c_{1}|1\rangle\left|\alpha e^{i \phi}\right\rangle_{b} .
$$

The state of the field $a$ is unaffected by the interaction, while the state of the field $b$ receives a phase shift, which is proportional to the number of photons on the the state $|\psi\rangle_{a}$.

Assume, we have two polarization qubits to become entangled. The qubits are initially prepared in singlephoton superposition states $|\psi\rangle_{a}=c_{0}|H\rangle+c_{1}|V\rangle$ and $|\psi\rangle_{b}=d_{0}|H\rangle+d_{1}|H\rangle$, where $|V\rangle$ and $|H\rangle$ are polarization degrees of freedom. These qubits are split individually on polarizing beam splitters into spatial modes and interact with an additional probe beam (which is in a coherent state $|\alpha\rangle_{p}$ ) in the Kerr medium. The resulting state of the three beams is given by

$$
\begin{aligned}
& |\psi\rangle_{a b c}=\left(c_{0} d_{0}|H H\rangle+c_{1} d_{1}|V V\rangle\right)|\alpha\rangle_{p} \\
& \quad+c_{0} d_{1}|H V\rangle\left|\alpha e^{i \phi}\right\rangle_{p}+c_{1} d_{0}|V H\rangle\left|\alpha e^{-i \phi}\right\rangle
\end{aligned}
$$

The first term in this expression does not receive any phase shift, while the the second and the third terms receive opposite sign shifts. This makes possible to transform the three-party state into entangled (Bell) bipartite states by performing a homodyne measurement on the probe. The measurement results into either $c_{0} d_{0}|H H\rangle+c_{1} d_{1}|V V\rangle$ or $c_{0} d_{1}|H V\rangle+c_{1} d_{0}|V H\rangle$ states, which are both maximally entangled states of qubits for $c_{0}=c_{1}=d_{0}=d_{1}=1 / \sqrt{2}$. It is also important to note that the described above Nemoto-Munro protocol allows us to construct entangling Controlled-NOT gate [7] with large Kerr nonlinearity, opening prominent possibility to use metamaterials in quantum computing.
Presented scheme for entanglement creation with negative index metamaterials may also find its applications in quantum communication and quantum teleportation with both coherent [5, 30] and polarization states [7]. Moreover, Kerr nonlinearity, created with the described medium, can be used to generate multimode entangled coherent states 32 and multiphoton Greenberger-HorneZeilinger states [33].

We also would like to outline that in the present discussion we restricted ourselves with TM polarization of surface polaritons. As we mentioned before, both TM and TE polarizations may exist on the dielectricmetamaterial interface. These polarizations may be used for information encoding on a par with encoding in quantum states of the field quanta. Another attractive idea is to use a trade-off between confinement and losses of the surface polaritons [22, 27]. This trade-off may be used to establish two regimes, corresponding to "manipulation" and "low-loss transmission", which are highly desired in quantum computation [34]. Both mentioned possibilities will be the subject of further investigations.

In conclusion, we presented the scheme for entanglement creation with the composite medium consisting of the dielectric and the negative index metamaterial. The surface polaritons, which are created by the incident light in the medium, propagate along the dielectricmetamaterial interface with substantially reduced group velocity, exhibiting property of spatial confinement and with suppressed amplitude losses. Placing a layer of six level atoms near the interface allowed us to establish symmetric nonlinear interaction between the surface polaritons, which can be utilized to create entanglement between initially uncorrelated coherent or polarization states of light.
[1] R. Horodecki et al., Rev. Mod. Phys. 81, 865 (2009).

[2] O. Gühne and G. Tóth, Phys. Rep. 474, 1 (2009).

[3] L. Amico et al., Rev. Mod. Phys. 80, 517 (2008).

[4] O. Romero-Isart et al., New J. Phys. 12, 033015 (2010).

[5] S.L. Braunstein and P. van Loock, Rev. Mod. Phys. 77, 513 (2005).

[6] B.C. Sanders, arXiv:1112.1778 (2011).

[7] P. Kok et al., Rev. Mod. Phys. 79, 135 (2007).

[8] M.D. Lukin and A. Imamoğlu, Phys. Rev. Lett. 84, 1419 (2000).

[9] D. Petrosyan and G. Kurizki, Phys. Rev. A 65, 033833 (2002).

[10] Z.-B. Wang, K.-P. Marzlin and B.C. Sanders, Phys. Rev. Lett. 97, 063901 (2006).

[11] D.D. Yavuz and D.E. Sikes, Phys. Rev. A 81, 035804 (2010).

[12] H. Kang and Y. Zhu, Phys. Rev. Lett. 91, 093601 (2003).

[13] Y.-F. Chen et al., Phys. Rev. Lett. 96, 043603 (2006).

[14] S. Li et al., Phys. Rev. Lett. 101, 073602 (2008).
[15] J.H. Shapiro, Phys. Rev. A 73, 062305 (2006).

[16] J. Gea-Banacloche, Phys. Rev. A 81, 043823 (2010).

[17] S.A. Maier, Plasmonics: Fundamentals and Applications (Springer, 2007).

[18] R. Merlin, Proc. Nat. Acad. Sci. 106, 1693 (2009).

[19] A.A. Kamli, S.A. Moiseev and B.C. Sanders, Phys. Rev. Lett. 101, 263601 (2008).

[20] M. Fleischhauer, A. Imamoğlu and J.P. Marangos, Rev. Mod. Phys. 77, 633 (2005)

[21] V.M. Shalaev, Nature Photonics 1, 41 (2007).

[22] A.A. Kamli, S.A. Moiseev and B.C. Sanders, Int. J. Quant. Inf. 9, 263 (2011).

[23] M.O. Scully and M.S. Zubairy, Quantum Optics (Cambridge University Press, 1997).

[24] N.A.R. Bhat and J.E. Sipe, Phys. Rev. A 73, 063808 (2006).

[25] P.Y. Chen et al., Phys. Rev. A 82, 053825 (2010).

[26] P. Ginzburg and A.V. Zayats, Opt. Expr. 20, 6720 (2012). 
[27] S.A. Moiseev, A.A. Kamli and B.C. Sanders, Phys. Rev. A 81, 033839 (2010).

[28] K.-P. Marzlin et al., J. Opt. Soc. Am. B 27, A36 (2010).

[29] M. Paternostro, M.S. Kim and B.S. Ham, Phys. Rev. A 67, 023811 (2003).

[30] S.J. van Enk and O. Hirota, Phys. Rev. A 64, 022313 (2001).
[31] K. Nemoto and W.J. Munro, Phys. Rev. Lett. 93, 250502 (2004).

[32] S.J. van Enk, Phys. Rev. Lett. 91, 017902 (2003).

[33] G.-S. Jin, Y. Lin and B. Wu, Phys. Rev. A 75, 054302 (2007).

[34] T.D. Ladd et al., Nature 464, 45 (2010). 